\title{
Multicenter Study of Antibiotic Resistance Profile of H. pylori and Distribution of CYP2C19 Gene Polymorphism in Rural Population of Chongqing, China
}

\author{
Ran Han, ${ }^{1}$ Hong Lu, ${ }^{2}$ Ming-Wan Jiang, ${ }^{3}$ Ke-Wen Tan, ${ }^{4}$ Zhong Peng, ${ }^{5}$ Jia-Li Hu, ${ }^{6}$ \\ Dian-Chun Fang, ${ }^{6}$ Chun-Hui Lan, ${ }^{7}$ and Xiao-Ling $\mathrm{Wu}^{1}$ \\ ${ }^{1}$ The Second Affiliated Hospital of Chongqing Medical University, Chongqing 400016, China \\ ${ }^{2}$ Department of Gastroenterology, Changshou District People's Hospital, Chongqing 400016, China \\ ${ }^{3}$ Department of Gastroenterology, Sanxia Central Hospital, Chongqing 400016, China \\ ${ }^{4}$ Department of Gastroenterology, Dianjiang County People's Hospital, Chongqing 400016, China \\ ${ }^{5}$ Department of Gastroenterology, Qijiang County People's Hospital, Chongqing 400016, China \\ ${ }^{6}$ Department of Gastroenterology, Southwest Hospital, Third Military Medical University, Chongqing 400016, China \\ ${ }^{7}$ Department of Gastroenterology, Daping Hospital, Third Military Medical University, Chongqing 400042, China
}

Correspondence should be addressed to Chun-Hui Lan; tiandaochouqin99@hotmail.com

and Xiao-Ling Wu; wuxiaoling@cqmu.edu.cn

Received 21 January 2016; Revised 5 April 2016; Accepted 11 April 2016

Academic Editor: Daniele Marrelli

Copyright (C) 2016 Ran Han et al. This is an open access article distributed under the Creative Commons Attribution License, which permits unrestricted use, distribution, and reproduction in any medium, provided the original work is properly cited.

\begin{abstract}
This study was to investigate the antibiotic resistance profile of $H$. pylori and the distribution of CYP2C19 gene polymorphism in rural population of Chongqing, China. 214 and 111 strains of $H$. pylori were isolated from rural and urban patients, respectively. $99.53 \%, 20.09 \%$, and $23.36 \%$ of the isolates in rural patients were found to be resistant to metronidazole, clarithromycin, and levofloxacin, while the resistant rate in urban patients was $82.88 \%, 19.82 \%$, and $24.32 \%$. The multiple antibiotic resistance percentage significantly increased from $28.26 \%$ (below 45 years) to $41.80 \%$ (above 45 years) in rural patients. Up to $44.39 \%$, $45.79 \%$, and $9.81 \%$ of rural patients from whom $H$. pylori was isolated were found to be extensive metabolizers, intermediate metabolizers, and poor metabolizers. No correlation was observed between antibiotic resistance profile of $H$. pylori and genetic polymorphism of CYP2C19 among rural population. There was a high prevalence of $H$. pylori strains resistant to metronidazole, clarithromycin, and levofloxacin in rural patients in Chongqing, China. The choice of therapy in this area should be based on local susceptibility patterns. Amoxicillin, gentamicin, and furazolidone are recommended as the first-line empiric regimen.
\end{abstract}

\section{Introduction}

Helicobacter pylori is a gram-negative, helical, microaerophilic bacterium that colonizes the gastric mucosa of approximately $50 \%$ of the world's population [1]. The prevalence of $H$. pylori infection ranges from $25-50 \%$ in the developed countries to over $80 \%$ in the developing world. In China, an estimated 40 to $60 \%$ of population is infected by $H$. pylori, with an average infection rate of $54.76 \%$ [2]. Race, gender, age, geographical location, and socioeconomic status are known to be associated with the prevalence of $H$. Pylori infection. The organism has been implicated in the pathogenesis of gastric disorders such as chronic gastritis, peptic ulcer disease, gastric carcinoma, and gastric lymphoma [3, 4]. Epidemiological studies have demonstrated an association between $H$. pylori infection and an increased risk of gastric cancer [5]. According to a meta-analysis, $H$. pylori infection was found to be associated with a 2 -fold increase in the risk for gastric cancer [6]. In a prospective study, approximately $2.9 \%$ of the patients infected with $H$. pylori eventually developed gastric cancer, while none of the patients without $H$. pylori infection developed gastric cancer [7]. At least $75 \%$ of new gastric cancer cases every year are thought to be linked to $H$. pylori infection [8]. It has been suggested that widespread treatment 
and elimination of $H$. pylori infection could protect against the occurrence of gastric cancer in people with no precancerous lesions $[9,10]$.

So far, triple therapy comprising a combination of two antibiotics (amoxicillin, clarithromycin, tinidazole, or metronidazole) and one proton pump inhibitor (PPI) (omeprazole, lansoprazole, pantoprazole, or rabeprazole) has yielded the best results in the treatment of H. pylori infection. However, with the recent emergence of antibiotic resistant strains of $H$. pylori, the efficacy of the standard triple therapy has reportedly decreased to $<80 \%$ [11]. A retrospective analytic study revealed a progressive decrease in the $H$. pylori eradication rate achieved with the standard triple therapy (metronidazole, clarithromycin, and amoxicillin) in China, from $88.56 \%$ prior to year 2004, to $77.66 \%$ between 2005 and 2009 , and further down to $71.13 \%$ from the year 2010 onwards [12]. Given the large population and the vast geographical expanse of China, the use of antibiotics for treatment of $H$. pylori infection is liable to vary greatly. Till date, the antibiotic resistance profile of $H$. pylori in the underdeveloped areas of China has not been documented. Thus, it is imperative to monitor antibiotic resistance of $H$. pylori in rural populations.

Proton pump inhibitors (PPIs) are known to be extensively metabolized in the liver by cytochrome P450 2C19 (CYP2C19). Although PPIs play an important role in improving the therapeutic efficacy of antibiotics for treatment of $H$. pylori infection, CYP2C19 gene polymorphism tends to affect both the pharmacokinetic and pharmacodynamic properties of PPIs. The genetic polymorphism of CYP2C19 is believed to be one of the major factors affecting the antibiotic resistance among $H$. pylori strains. Based on the ability to metabolize CYP2C19 substrates, three distinct phenotypes of CYP2C19 are known to exist: extensive metabolizers (EMs), intermediate metabolizers (IMs), and poor metabolizers (PMs) [13]. The distribution of CYP2C19 genetic polymorphism in the rural population of Chongqing region has not been investigated. This study represents the first systematic assessment of the antibiotic resistance profile of $H$. pylori and genetic polymorphism of CYP2C19 among the rural population in Chongqing, China. The objective is to provide a theoretical basis for the individualized therapy for H. pylori infection.

\section{Materials and Methods}

2.1. Study Subjects. The study was conducted from January 2015 to June 2015. The protocol was reviewed and approved by the Hospital Ethics Review Committee, Chongqing, China (no. (2014) 20). Subjects residing in rural areas of Chongqing region and presenting with abdominal pain, bloating, nausea, vomiting, hematemesis, or melena $(N=667)$ were recruited at 11 hospitals (Daping Hospital, Changshou District People's Hospital, Dianjiang County People's Hospital, Sanxia Central Hospital, Qianjiang Central Hospital, Qijiang County People's Hospital, Fengdu County People's Hospital, Dazu County People's Hospital, Fuling Central Hospital, Kaixian People's Hospital, and Hechuan District People's Hospital).

The inclusion criteria were as follows: (1) age: $18-75$ years, male or female; (2) being positive for ${ }^{13} \mathrm{C} /{ }^{14} \mathrm{C}$-urea breath test and $H$. pylori culture; (3) no history of treatment for
H. pylori infection; (4) no prior administration of antibiotics, bismuth, probiotics, $\mathrm{H} 2$ receptor antagonists, or PPIs in the immediately preceding 4 weeks; (5) residing in rural areas of Chongqing. Exclusion criteria were as follows: (1) patients treated with gastrectomy, gastric angioplasty, or vagus nerve amputation; (2) pregnant or lactating women; (3) patients with serious liver, kidney, heart, brain, lung, endocrine, or hematopoietic disorders; (4) patients with malignant tumors; (5) patients with Zollinger-Ellison syndrome; (6) participation in other clinical studies in the last three months.

Urban patients infected with $H$. pylori $(N=290)$ were also recruited during the same period from Daping Hospital, employing the same inclusion criteria except that the subjects were required to have always been living in urban areas in Chongqing. Written informed consent was obtained from all study subjects prior to their enrolment in the study.

2.2. Specimen Collection. All patients were subjected to upper gastrointestinal endoscopy (UGIE). For isolation of $H$. pylori, two gastric tissue specimens were collected, one from the antrum $<5 \mathrm{~cm}$ from the pylorus and the other from the gastric body. In addition, one gastric tissue specimen was collected from antrum for analysis of CYP2C19 gene polymorphism. All specimens were stored at $-80^{\circ} \mathrm{C}$ until further processing.

2.3. Isolation of $H$. pylori. The presence of $H$. pylori in the gastric tissue samples was determined on microbiological culture. Biopsies were ground and cultured on solid selective, enriched medium (H. pylori agar, Becton Dickinson) and incubated microaerophilically at $37^{\circ} \mathrm{C}$ for $72 \mathrm{~h}$. Transparent colonies were picked and subjected to gram staining to demonstrate the presence of gram-negative spiral or $\mathrm{S}$ shape bacteria. Isolates found to be positive for urease, catalase, and oxidase were considered as $\mathrm{H}$. pylori.

2.4. Drug Susceptibility Testing (DST). Drug susceptibility testing of $\mathrm{H}$. pylori isolates was performed by agar dilution method. The different antibiotic solutions with desired concentrations were added to Columbia agar medium containing $5 \%$ fresh defibrinated sheep blood before being poured into plates. Fresh colonies were picked with inoculating loop and formulated into bacterial suspension with sterile saline. Two microlitres of mixture was inoculated on plates containing antibiotics and incubated microaerophilically at $37^{\circ} \mathrm{C}$ for $72 \mathrm{~h}$. Growth in the presence of drug was regarded as being indicative of resistance, while absence of growth was considered indicative of susceptibility of the strain to that antibiotic. As per the US Clinical and Laboratory Standards Institute guidelines [14], the minimum inhibitory drug concentrations were as follows: metronidazole $8 \mu \mathrm{g} / \mathrm{mL}$, clarithromycin $1 \mu \mathrm{g} / \mathrm{mL}$, amoxicillin $2 \mu \mathrm{g} / \mathrm{mL}$, gentamicin $8 \mu \mathrm{g} / \mathrm{mL}$, levofloxacin $2 \mu \mathrm{g} / \mathrm{mL}$, and furazolidone $2 \mu \mathrm{g} / \mathrm{mL}$. NCTC11637 $\mathrm{H}$. pylori strain (43504, ATCC, USA) was used as the control strain. All the tests were conducted in Hangzhou Zhiyuan Medical Inspection Institute, China.

2.5. CYP2C19 Gene Polymorphism. Genomic DNA was extracted from stomach tissues using DNA extraction kit (Promega, USA) and subjected to $1 \%$ agarose 
TABLE 1: Results of drug susceptibility tests of $H$. pylori among the rural and urban population in Chongqing.

\begin{tabular}{|c|c|c|c|c|}
\hline \multirow{2}{*}{ Antibiotics } & \multicolumn{2}{|c|}{ Antibiotic resistant cases $(n)$ and rates (\%) } & \multirow{2}{*}{$\chi^{2}$} & \multirow{2}{*}{$P$ value } \\
\hline & Rural & Urban & & \\
\hline Metronidazole & $213(99.53)$ & $92(82.88)$ & 35.084 & 0.000 \\
\hline Clarithromycin & $43(20.09)$ & $22(19.82)$ & 0.003 & 0.953 \\
\hline Levofloxacin & $50(23.36)$ & $27(24.32)$ & 0.037 & 0.847 \\
\hline Amoxicillin & 0 & 0 & & \\
\hline Furazolidone & 0 & 0 & & \\
\hline Gentamicin & 0 & 0 & & \\
\hline
\end{tabular}

gel electrophoresis. Two polymerase chain reactions (CYP2C19*2 and CYP2C19*3) were performed using genomic DNA extracted from gastric mucosa as a template. The primer sequences were as follows: $5^{\prime}$-GCAGGTATAAGTCTAGGAAATG- $3^{\prime}$ and $5^{\prime}$-TAAAGTCCCGAGGGTTGTTG-3' for CYP2C19*2; 5'-CACCCTGTGATCCCACTTTC- $3^{\prime}$ and $5^{\prime}$-CTAATGGGCTTAGAAGCCTG-3' for CYP2C19*3. For $25 \mu \mathrm{L}$ PCR reaction, $1.5 \mu \mathrm{L}$ of DNA sample was mixed with $2.5 \mu \mathrm{L} 10 \mathrm{x}$ Master Mix, $1.25 \mu \mathrm{L} 10 \mu \mathrm{M}$ of forward and reverse primers each, $2 \mu \mathrm{L} 10 \mathrm{mM}$ dNTP, $1.5 \mu \mathrm{L}$ DNA Taq polymerase, and $15 \mu \mathrm{L}$ reverse transcriptase (RT-) PCR grade water. The PCR conditions used were as follows: initial denaturation at $94^{\circ} \mathrm{C}$ for $5 \mathrm{~min}$, followed by 30 amplification cycles, each consisting of denaturation at $94^{\circ} \mathrm{C}$ for $30 \mathrm{~s}$, annealing at $50^{\circ} \mathrm{C}$ for $30 \mathrm{~s}$, extension at $72^{\circ} \mathrm{C}$ for $1 \mathrm{~min}$, and final extension at $72^{\circ} \mathrm{C}$ for $5 \mathrm{~min}$. PCR product was recovered by PCR recovery kit (Tiangen, China).

CYP2C19*2 amplification products were sequenced using forward primers, while CYP2C19*3 amplification products were sequenced using reverse primers. The sequencing results were analyzed using Chromas and Sequencing Analysis 5.2 software. Based on the mutations on CYP2C19*2 and CYP2C19*3, patients were categorized as belonging to one of three groups: EM (636GG, 681GG), IM (636GG, 681GA), (636AG, 681GG), and PM (636GG, 681AA), (636AG, 681AG), (636AA, 681GG).

2.6. Statistical Analysis. Chi-square test was used to assess potential association of antibiotic resistance profile of $H$. pylori with the following variables: gender, age, body mass index (BMI), disease type, housing area, education status, source of drinking water, smoking, and alcohol intake, as well as the CYP2C19 phenotypes. $P<0.05$ was considered statistically significant. All statistical analyses were performed using SPSS 16.0 software.

\section{Results}

3.1. Antibiotic Resistance Profile of H. pylori in Rural and Urban Populace. 214 strains were isolated from rural patients, with a mean age of $47.6 \pm 10.1,112$ male and 102 female. 111 strains were isolated from urban patients, with a mean age of $46.8 \pm 9.8,70$ male and 41 female.

Table 1 showed the antibiotic resistance profile of $H$. pylori isolates to six antibiotics. In rural patients, $99.53 \%$, $20.09 \%$, and $23.36 \%$ isolates were found to be resistant
TABle 2: Correlation between $H$. pylori antibiotic resistance and CYP2C19 genetic polymorphism in rural patients.

\begin{tabular}{lccc}
\hline CYP2C19 & \multicolumn{3}{c}{ Antibiotic resistant cases $(n)$ and rates (\%) } \\
phenotype & Clarithromycin & Levofloxacin & Metronidazole \\
\hline EMs & $21(22.11)$ & $17(17.89)$ & $95(100)$ \\
IMs & $16(16.33)$ & $20(20.41)$ & $97(98.98)$ \\
PMs & $6(28.57)$ & $3(14.29)$ & $21(100)$ \\
$P$ value & 0.360 & 0.780 & NS \\
\hline
\end{tabular}

EMs, extensive metabolizers; IMs, intermediate metabolizers; PMs, poor metabolizers.

NS: not significant by chi-square test.

to metronidazole, clarithromycin, and levofloxacin, respectively, while none of the isolates were resistant to amoxicillin, furazolidone, or gentamicin. Resistance to metronidazole + clarithromycin/levofloxacin was found in $28.5 \%$ isolates, while that to metronidazole + clarithromycin + levofloxacin was found in $7.5 \%$ isolates. In urban patients, $82.88 \%, 19.82 \%$, and $24.3 \%$ of all isolates were found to be resistant to metronidazole, clarithromycin, and levofloxacin, respectively. Resistance to metronidazole + clarithromycin/levofloxacin was found in $13.51 \%$ isolates, while that to metronidazole + clarithromycin + levofloxacin was found in $15.32 \%$ isolates. The resistance rate to metronidazole in rural patients was significantly higher than that in urban patients $(P<0.05)$.

3.2. Correlation of $H$. pylori Multiple Antibiotic Resistance and the Characteristics of Rural Patients. No significant correlation was observed between antibiotic resistance among H. pylori isolates and rural patient characteristics such as gender, body mass index (BMI), disease type, housing area, education status, source of drinking water, smoking, and alcohol intake, except age. The multiple antibiotic resistance percentage increased from $28.26 \%$ (below 45 years) to $41.80 \%$ (above 45 years) $\left(\chi^{2}=4.176, P<0.05\right)$.

3.3. Correlation between H. pylori Antibiotic Resistance and CYP2C19 Genetic Polymorphism in Rural Patients. Chisquare test was used to assess differences in the antibiotic resistance profile of $H$. pylori isolated from rural patients with different CYP2C19 phenotypes. No significant correlation was observed between CYP2C19 phenotypes and resistance of $H$. pylori isolates to clarithromycin, levofloxacin, and metronidazole (Table 2). 
3.4. Prevalence of CYP2C19 Phenotype in Rural and Urban Patients. The phenotype and frequency of CYP2C19 alleles in rural and urban patients from whom the $H$. pylori strains were isolated were compared by chi-square test. EMs, IMs, and PMs accounted for $44.39 \%, 45.79 \%$, and $9.81 \%$ of positive patients in rural areas and $33.33 \%, 54.96 \%$, and $11.71 \%$ in urban patients. No statistically significant difference was found in the frequency of CYP2C19 phenotypes between rural and urban patients $\left(\chi^{2}=3.706, P>0.05\right)$.

\section{Discussion}

H. pylori is one of the most common human pathogens with a global infection rate of $>50 \%$. The prevalence of $H$. pylori infection in adult population of China is estimated to be $40-60 \%$ [2]. Treatment of $H$. pylori infection by a triple or quadruple drug regime is recommended; however, the emergence of antibiotic resistance is an important issue to be considered $[15,16]$. Globally, a rising trend in the emergence of resistance to metronidazole, clarithromycin, and levofloxacin among $H$. pylori isolates has been acknowledged [17]. With the widespread use of antibiotics for treatment of $H$. pylori infection, the phenomenon of antibiotic resistance is expected to gradually increase. Indeed, $60 \%$ to $70 \%$ isolates of $H$. pylori are reportedly resistant to metronidazole [18].

A prospective study conducted in Beijing reported 66.8\% and $63.4 \%$ prevalence of resistance to metronidazole among $H$. pylori isolates in years 2009-2010 and 2013-2014, respectively [19]. In the present study, $99.6 \% \mathrm{H}$. pylori isolates from rural patients were found to be resistant to metronidazole, which was significantly higher than that reported from other parts of China and abroad [20, 21]. This is likely related to the misuse of antibiotics and the generally lax control over the sale of antibiotics. However, further epidemiological investigations are required to identify the specific reasons.

In view of the results of our study, metronidazole should not be used for treatment of $H$. pylori infection, particularly in the rural areas of Chongqing. Clarithromycin is one of the main components of the standard triple therapy for treatment of $H$. pylori infection. The increased resistance to clarithromycin is liable to directly impact on the eradication rates of $H$. pylori [22]. In the present study, 20.1\% of $H$. pylori isolates were resistant to clarithromycin, which is in line with that reported in a recent report [23].

According to the Maastricht IV/Florence Consensus Report [24], if $>15-20 \%$ isolates of $H$. pylori develop resistance to clarithromycin, nonbismuth quadruple therapy should be avoided. Therefore, in rural areas of Chongqing, clarithromycin should only be prescribed based on the results of drug susceptibility testing. The resistance rate to levofloxacin was found to be $22.2 \%$, which is consistent with the estimated national prevalence [25]. None of the H. pylori isolates were found to be resistant to amoxicillin, furazolidone, and gentamicin. These three drugs may be used in the absence of data on drug susceptibility.

Antibiotic resistance among $H$. pylori strains manifests considerable geographical variations [26]. In the present study, we not only investigated the resistance rate in patients from rural areas in Chongqing, but also demonstrated a lack of correlation between the $H$. pylori antibiotic resistance and gender, body mass index (BMI), disease type, housing area, education status, source of drinking water, smoking, and/or alcohol intake, except age. Resistance to multiple antibiotics is more likely in patients over the age of 45 than those less than 45 years $(P<0.05)$. Guo et al. [27] demonstrated that the antibiotic resistance rate of $H$. pylori increased with age, which might be due to the antibiotic accumulation. Our findings suggest the relevance of the notion of individualized medicine in the clinical treatment of $H$. pylori, especially in the rural population, where a $28.5 \%$ prevalence of resistance to double drugs (metronidazole + clarithromycin/ levofloxacin) and $7.5 \%$ prevalence to triple drug therapy (metronidazole + clarithromycin + levofloxacin) were observed.

Several reasons are attributed to bacterial resistance to antibiotics, such as frequent consumption of antibiotics, so that the resistant bacteria survive the harsh environment and could then spread the resistance genes, an intrinsic property or an occurring mutation in the chromosomal genes, such as CYP2C19. CYP2C19 is one of the most important drugmetabolizing enzymes belonging to CYP450 family and is mainly found in microsomes of hepatocytes. On the basis of their ability to metabolize CYP2C19 substrates, individuals can be classified as extensive metabolizers (EMs), intermediate metabolizers (IMs), or poor metabolizers (PMs) [13]. Genetic polymorphism (mainly CYP2C19*2, CYP2C19*3, and CYP2C19*17) exists for CYP2C19 expression [28]. Two common mutant alleles of the CYP2C19 gene among the Asian population are CYP2C19*2 and CYP2C19*3 [29].

Prevalence of CYP2C19 gene polymorphism varies in different ethnic groups [30]. EMs are known to account for a vast majority of Europeans, while in the Asian population, PMs and EMs constitute a significant proportion. This phenomenon was believed to lead to marked individual differences in drug efficacy [31]. CYP2C19 mutations could influence the stomach $\mathrm{pH}$ value and thus affect the activity and stability of antibiotics, such as amoxicillin and clarithromycin [32]. Bacteria are not destroyed by these antibiotics which are not fully active in an acid environment but are exposed to subinhibitory concentrations of drugs for many hours. This might lead to the microevolution of bacteria and the appearance of resistant strains.

The present study showed that the distribution of CYP2C19 gene polymorphism in the rural population in Chongqing is in agreement with that of the Asian population [33]. No significant difference in CYP2C19 gene polymorphism was found between urban and rural populations of Chongqing. Moreover, genetic polymorphism of CYP2C19 was not found to influence antibiotic resistance profile of $H$. pylori isolated from rural population. This is the first study to investigate antibiotic resistance of $H$. pylori and distribution of CYP2C19 gene polymorphism in rural population in Chongqing.

In summary, due to the high prevalence of $H$. pylori strains resistant to metronidazole, clarithromycin, and levofloxacin in rural patients, the choice of therapy in this area should be based on local susceptibility patterns. Metronidazole should not be used for the treatment of $H$. pylori 
infection in the rural areas of Chongqing, China. Amoxicillin, gentamicin, and furazolidone are recommended as the firstline empiric therapy regimens.

\section{Competing Interests}

The authors declare that they have no competing interests.

\section{Authors' Contributions}

Chun-Hui Lan and Xiao-Ling Wu contributed equally to this work; Chun-Hui Lan and Xiao-Ling Wu designed the protocol; Ran Han, Hong Lu, Ming-Wan Jiang, Ke-Wen Tan, Zhong Peng, and Jia-Li Hu performed the study; Dian-Chun Fang contributed reagents/analytic tools; Ran Han and Chun-Hui Lan analyzed the data; Ran Han, Chun-Hui Lan, and XiaoLing Wu wrote the paper.

\section{Acknowledgments}

The authors would like to thank Hong Peng, Hechuan District People's Hospital; Ming Wang, Dazu County People's Hospital; Ai-min Liu, Fuling Central Hospital; Tao Luo, Qianjiang Central Hospital; Zhi-Rong Ao, Fengdu County People's Hospital; and Jian Li, Kaixian People's Hospital, for their involvement in recruiting study subjects. This work was supported by grants from National Natural Science Foundation of China: (1) (no. 81472006) The Mechanism Underlying Gastric Epithelial Cell Death Induced by the Activation of NLRP3 Inflammatory Pathway by Helicobacter pylori VacA; (2) (no. 81171526) Cortactin/Actin Mediated the Transportation of Helicobacter pylori VacA to Mitochondria.

\section{References}

[1] P. Moayyedi and R. H. Hunt, "Helicobacter pylori public health implications," Helicobacter, vol. 9, supplement 1, pp. 67-72, 2004.

[2] W.-D. Zhang, F.-L. Hu, S.-D. Xiao, and Z.-M. Xu, "Prevalence of Helicobacter pylori infection in China," Modern Digestion \& Intervention, vol. 15, no. 5-6, pp. 265-270, 2010.

[3] D. Ertem, H. Harmanci, and E. Pehlivanoğlu, "Helicobater pylori infection in Turkish preschool and school children: role of socioeconomic factors and breast feeding," The Turkish Journal of Pediatrics, vol. 45, no. 2, pp. 114-122, 2003.

[4] E. Ierardi, E. Goni, G. Losurdo, and F. Di Mario, "Helicobacter pylori and nonmalignant diseases," Helicobacter, vol. 19, supplement 1, pp. 27-31, 2014.

[5] L. E. Wroblewski, R. M. Peek Jr., and K. T. Wilson, "Helicobacter pylori and gastric cancer: factors that modulate disease risk," Clinical Microbiology Reviews, vol. 23, no. 4, pp. 713-739, 2010.

[6] G. D. Eslick, L. L.-Y. Lim, J. E. Byles, H. H.-X. Xia, and N. J. Talley, "Association of Helicobacter pylori infection with gastric carcinoma: a meta-analysis," The American Journal of Gastroenterology, vol. 94, no. 9, pp. 2373-2379, 1999.

[7] N. Uemura, S. Okamoto, S. Yamamoto et al., "Helicobacter pylori infection and the development of gastric cancer," The New England Journal of Medicine, vol. 345, no. 11, pp. 784-789, 2001.

[8] C. De Martel, J. Ferlay, S. Franceschi et al., "Global burden of cancers attributable to infections in 2008: a review and synthetic analysis," The Lancet Oncology, vol. 13, no. 6, pp. 607-615, 2012.
[9] A. C. Ford, D. Forman, R. H. Hunt, Y. Yuan, and P. Moayyedi, "Helicobacter pylori eradication therapy to prevent gastric cancer in healthy asymptomatic infected individuals: systematic review and meta-analysis of randomised controlled trials," The British Medical Journal, vol. 348, Article ID g3174, 2014.

[10] C.-W. Lee, B. Rickman, A. B. Rogers, Z. Ge, T. C. Wang, and J. G. Fox, "Helicobacter pylori eradication prevents progression of gastric cancer in hypergastrinemic INS-GAS mice," Cancer Research, vol. 68, no. 9, pp. 3540-3548, 2008.

[11] D. Y. Graham and L. Fischbach, "Helicobacter pylori treatment in the era of increasing antibiotic resistance," Gut, vol. 59, no. 8, pp. 1143-1153, 2010.

[12] B. Wang, Z.-F. Lv, Y.-H. Wang et al., "Standard triple therapy for Helicobacter pylori infection in China: a meta-analysis," World Journal of Gastroenterology, vol. 20, no. 40, pp. 14973-14985, 2014.

[13] Z. Desta, X. Zhao, J.-G. Shin, and D. A. Flockhart, "Clinical significance of the cytochrome P450 2C19 genetic polymorphism," Clinical Pharmacokinetics, vol. 41, no. 12, pp. 913-958, 2002.

[14] Clinical and Laboratory Standards Institute, M100-s23 Performance Standards for Antimicrobial Susceptibility Testing: Twenty-Third Informational Supplement, CLSI, Wayne, Pa, USA, 2013.

[15] F. Khademi, J. Faghri, F. Poursina et al., "Resistance pattern of Helicobacter pylori strains to clarithromycin, metronidazole, and amoxicillin in Isfahan, Iran," Journal of Research in Medical Sciences, vol. 18, no. 12, pp. 1056-1060, 2013.

[16] P. H. Baglan, G. Bozdayi, M. Ozkan, K. Ahmed, A. M. Bozdayi, and A. Ozden, "Clarithromycin resistance prevalence and Icea gene status in Helicobacter pylori clinical isolates in Turkish patients with duodenal ulcer and functional dyspepsia," Journal of Microbiology, vol. 44, no. 4, pp. 409-416, 2006.

[17] V. De Francesco, F. Giorgio, C. Hassan et al., "Worldwide H. pylori antibiotic resistance: a systematic review," Journal of Gastrointestinal and Liver Diseases, vol. 19, no. 4, pp. 409-414, 2010.

[18] GROUP C H P R, "Prevalence of Helicobacter pylori research to antibiotics and its influence on the treatment outcome in China:a multicenter clinical study," Chinese Journal of Gastroenterology, vol. 12, no. 9, pp. 525-530, 2007.

[19] Y.-X. Zhang, L.-Y. Zhou, Z.-Q. Song, J.-Z. Zhang, L.-H. He, and Y. Ding, "Primary antibiotic resistance of Helicobacter pylori strains isolated from patients with dyspeptic symptoms in Beijing: a prospective serial study," World Journal of Gastroenterology, vol. 21, no. 9, pp. 2786-2792, 2015.

[20] A. Talebi Bezmin Abadi, A. Ghasemzadeh, T. Taghvaei, and A. M. Mobarez, "Primary resistance of Helicobacter pylori to levofloxacin and moxifloxacine in Iran," Internal and Emergency Medicine, vol. 7, no. 5, pp. 447-452, 2012.

[21] P. Kostamo, L. Veijola, A. Oksanen, S. Sarna, and H. Rautelin, "Recent trends in primary antimicrobial resistance of Helicobacter pylori in Finland," International Journal of Antimicrobial Agents, vol. 37, no. 1, pp. 22-25, 2011.

[22] J. Molina-Infante and J. P. Gisbert, “Optimizing clarithromycincontaining therapy for Helicobacter pylori in the era of antibiotic resistance," World Journal of Gastroenterology, vol. 20, no. 30, pp. 10338-10347, 2014.

[23] V. Papastergiou, S. D. Georgopoulos, and S. Karatapanis, "Treatment of Helicobacter pylori infection: meeting the challenge of antimicrobial resistance," World Journal of Gastroenterology, vol. 20, no. 29, pp. 9898-9911, 2014. 
[24] P. Malfertheiner, F. Megraud, C. A. O’Morain et al., "Management of Helicobacter pylori infection-the maastricht IV/ florence consensus report," Gut, vol. 61, no. 5, pp. 646-664, 2012.

[25] F. L. Hu, "Antibiotic resistance status of H. pylori in China," Weichangbingxue He Ganbingxue Zazhi, vol. 17, pp. 517-518, 2008.

[26] J. Chen, F. B. Chen, J. D. Yu et al., "Prevalence of Helicobacter pylori resistant to clarithromycin, amoxicillin and metronidazole in children," Chinese Journal of Pediatrics, vol. 42, no. 10, pp. 769-771, 2004.

[27] F. Guo, F. H. Zhang, Q. Bi et al., "Analysis on antibiotic resistance of Helicobacter pylori strains isolated from deferent age groups in Xiaoshan area," Chinese Journal of Digestive Endoscopy, vol. 32, no. 9, pp. 631-632, 2015.

[28] L. Bertilsson, "Geographical/interracial differences in polymorphic drug oxidation. Current state of knowledge of cytochromes P450 (CYP) 2D6 and 2C19," Clinical Pharmacokinetics, vol. 29, no. 3, pp. 192-209, 1995.

[29] S. M. F. De Morais, G. R. Wilkinson, J. Blaisdell, K. Nakamura, U. A. Meyer, and J. A. Goldstein, "The major genetic defect responsible for the polymorphism of S-mephenytoin metabolism in humans," The Journal of Biological Chemistry, vol. 269, no. 22, pp. 15419-15422, 1994.

[30] A. E. Rettie and J. P. Jones, "Clinical and toxicological relevance of CYP2C9: drug-drug interactions and pharmacogenetics," Annual Review of Pharmacology and Toxicology, vol. 45, pp. 477-494, 2005.

[31] I. Ieiri, T. Kubota, A. Urae et al., "Pharmacokinetics of omeprazole (a substrate of CYP2C19) and comparison with two mutant alleles, CYP2C19 ${ }_{m 1}$ in exon 5 and CYP2C19 ${ }_{m 2}$ in exon 4 , in Japanese subjects," Clinical Pharmacology and Therapeutics, vol. 59, no. 6, pp. 647-653, 1996.

[32] P. O. Erah, A. F. Goddard, D. A. Barrett, P. N. Shaw, and R. C. Spiller, "The stability of amoxycillin, clarithromycin and metronidazole in gastric juice: relevance to the treatment of Helicobacter pylori infection," The Journal of Antimicrobial Chemotherapy, vol. 39, no. 1, pp. 5-12, 1997.

[33] K. Adachi, T. Katsube, A. Kawamura et al., "CYP2C19 genotype status and intragastric $\mathrm{pH}$ during dosing with lansoprazole or rabeprazole," Alimentary Pharmacology \& Therapeutics, vol. 14, no. 10, pp. 1259-1266, 2000. 


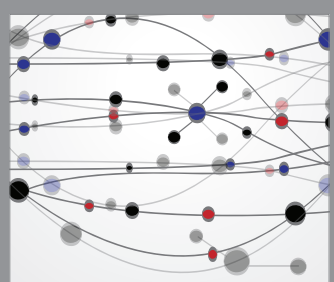

The Scientific World Journal
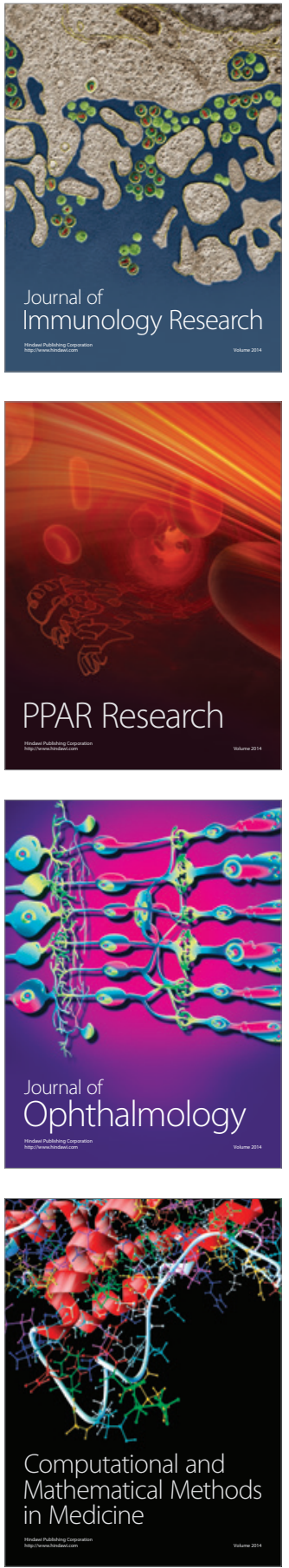

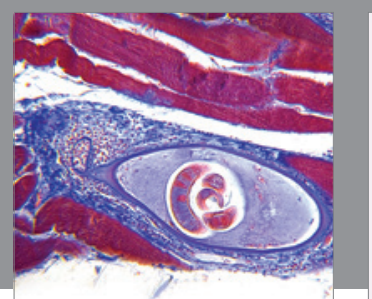

Gastroenterology Research and Practice

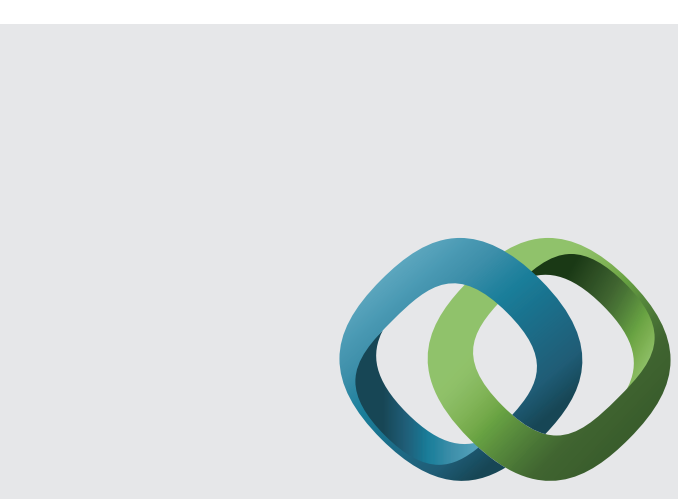

\section{Hindawi}

Submit your manuscripts at

http://www.hindawi.com
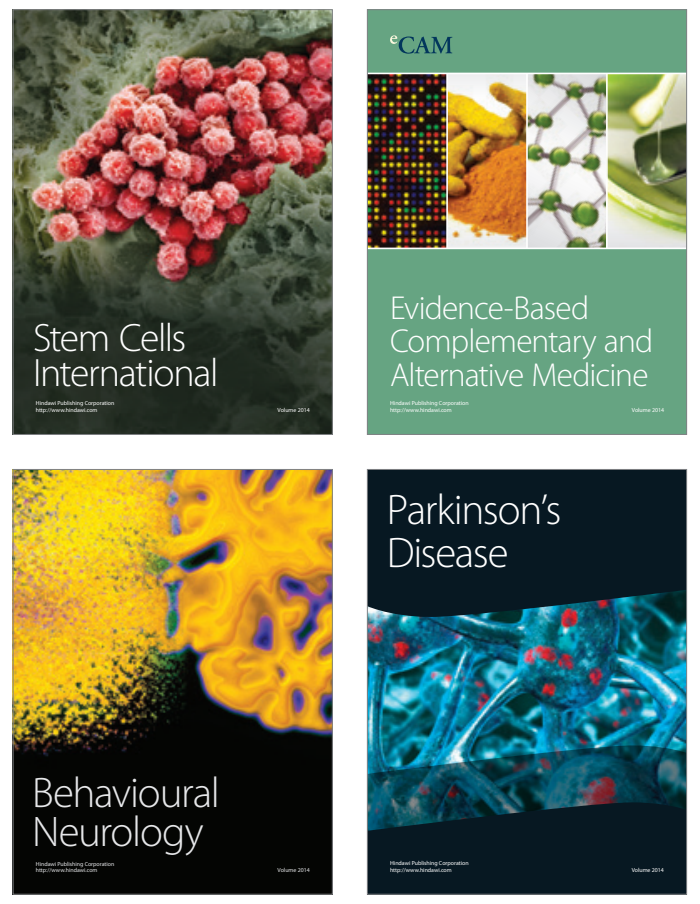
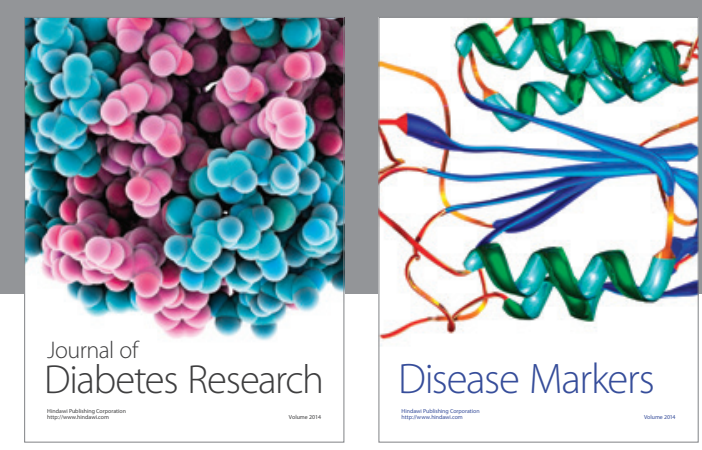

Disease Markers
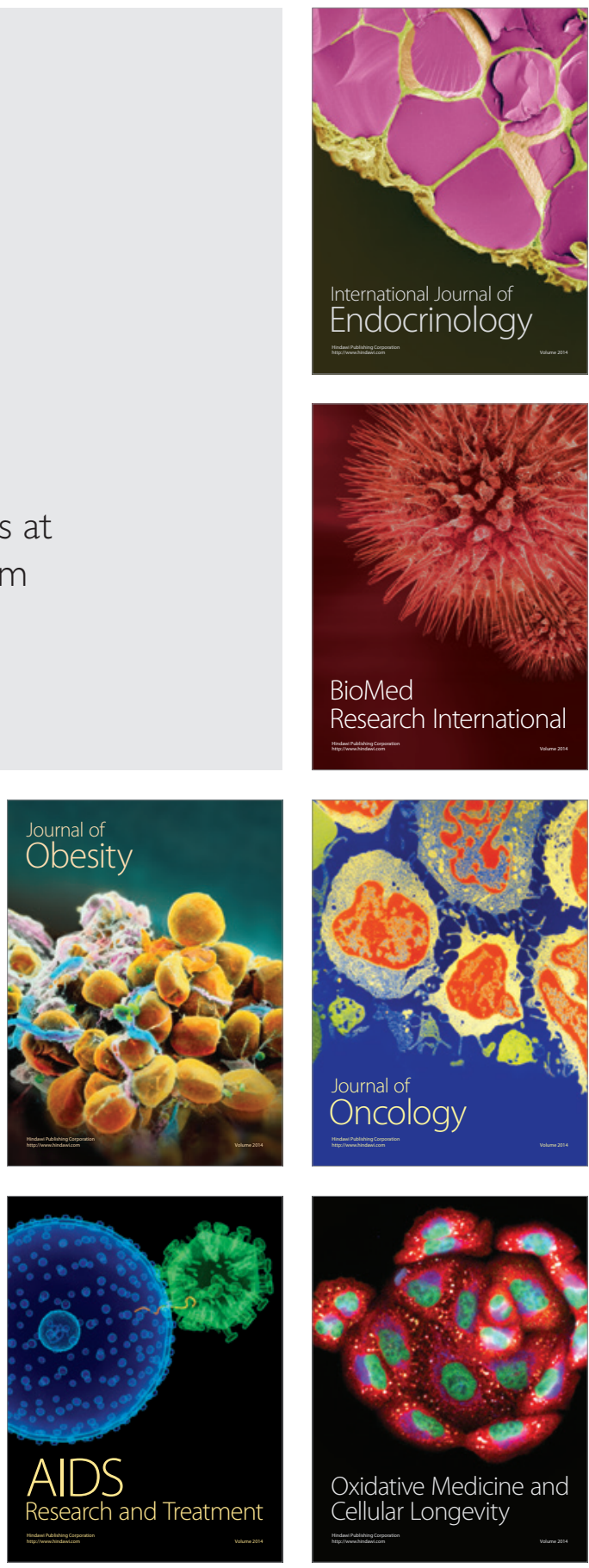